\section{Cognitive effects of lamotrigine versus topiramate as adjunctive therapy in older adults with epilepsy}

\author{
Steve S. Chung, ' Susan Kerls, ${ }^{2}$ \\ Ann Hammer, Robert Kustra, ${ }^{2}$ \\ 'Barrow Neurological Institute, USA; \\ ${ }^{2}$ Neuroscience Development Center, \\ GlaxoSmithKline, Research Triangle Park, \\ NC, USA; ${ }^{3}$ Neuroscience Development \\ Center, GlaxoSmithKline, Research \\ Triangle Park, NC, USA
}

\section{Abstract}

Older individuals may be more susceptible to cognitive side effects of antiepileptic drugs than are younger adults. This randomized, double-blind study compared the cognitive effects of lamotrigine (median maintenance dosage, $500.0 \mathrm{mg} / \mathrm{d}$ ) and topiramate (median maintenance dosage, $300.0 \mathrm{mg} / \mathrm{d}$ ) as adjunctive therapy for 16 weeks in patients $\geq 50$ years of age. Fifty-one patients (lamotrigine, $n=25$; topiramate, $\mathrm{n}=26$ ) were enrolled, and 28 patients (lamotrigine, $\mathrm{n}=15$; topiramate, $\mathrm{n}=13$ ) completed the study. In a combined analysis of all cognitive tests performed, no significant differences between treatment groups were noted. However, analyses of individual cognitive test results revealed that lamotrigine-treated patients had significantly better results on the Controlled Oral Word Association Test and the Symbol-Digit Modalities Test, whereas topiramate-treated patients had significantly more favorable results on the Digit Cancellation Test and the Rey Auditory-Verbal Learning Test. Larger studies are needed to further clarify the differences in the cognitive effects of lamotrigine and topiramate in older patients.

\section{Introduction}

In comparison to younger adults, older individuals tend to be more susceptible to cognitive side effects of antiepileptic drug (AED) therapy. ${ }^{1-4}$ Some cognitive functions, such as memory, may already be declining in older patients, which may make such patients less tolerant of cognitive side effects associated with AEDs. ${ }^{2,3}$ Additionally, age-related changes in AED metabolism and pharmacokinetics occur, such as decreased plasma protein binding, decreased renal drug clearance, decreased hepatic oxidation, and longer elimination halflife, ${ }^{2,5}$ which may influence patients' suscepti- bility to cognitive effects of AEDs. Older patients are also more likely to be receiving multiple medications for comorbid conditions, thereby increasing the possibility of drug interactions $^{1}$ and possibly affecting patients' susceptibility to cognitive side effects.

Cognitive effects differ between the various AEDs. Although there are no clinical studies comparing the cognitive side effects of AEDs in older patients with epilepsy, many comparative studies were performed in younger patients. For example, previous studies suggested that lamotrigine was less commonly associated with the cognitive impairment that was seen with many other AEDs and topiramate may pose cognitive side effects. ${ }^{6,7}$ Also the results from a more recent study which compared retention rates among newer AEDs suggested that drug discontinuations for wordfinding difficulties were more common with topiramate than with any other drug studied. ${ }^{8}$ In addition, a randomized, double-blind study by Blum et al. ${ }^{9}$ compared the cognitive effects of lamotrigine and topiramate administered as adjunctive therapy in adults with epilepsy, and the results showed greater cognitive impairment associated with topiramate therapy. However, it is not well understood whether older patients would experience different cognitive side effects than younger adults, and if so, how they would differ.

\section{Materials and Methods}

\section{Study participants}

To be eligible for study inclusion, patients were required to have had a confident diagnosis of partial epilepsy for $\geq 6$ months and to have had at least one complex partial or secondarily generalized tonic-clonic seizure (but no more than 8 seizures per month) in the past three months. This study included patients who are 50 years of age or older. Patients were also required to be receiving carbamazepine or phenytoin as monotherapy or in combination with one additional AED that is neither an enzyme inducer nor inhibiter and to have had no change of $>10 \%$ in AED dose for at least one month before study enrollment. Patients with previous exposure to lamotrigine or topiramate were excluded from the study. The protocol and informed consent were approved by Institutional Review Boards for each site. All patients provided written informed consent.

\section{Study design}

This randomized, double-blind, multicenter study began with a screening phase of $\leq 2$ weeks, during which patients were screened for eligibility and were administered a battery of cognitive tests to establish baseline scores.
Correspondence: Steve S. Chung,

Barrow Neurological Institute, USA

E-mail: sschung@chw.edu

Received for publication: 18 March 2009.

Revision received: 25 May 2009.

Accepted for publication: 11 June 2009.

Key words: cognitive side effects, lamotrigine, epilepsy, older patients.

This work is licensed under a Creative Commons Attribution 3.0 License (by-nc 3.0)

CCopyright S.S. Chung et al., 2009

Licensee PAGEPress, Italy

Neurology International 2009; 1:e6

doi:10.4081/ni.2009.e6

After the screening phase, patients were randomly assigned, in a 1:1 ratio, to receive either lamotrigine or topiramate as add-on therapy. Drug dosages were titrated to a target maintenance dosage during an 8-week dose escalation phase (Table 1). The dose escalation phase was followed by an 8-week maintenance phase, during which stable dosages of lamotrigine or topiramate and concomitant AEDs were maintained.

\section{Study end points}

The primary end point of the study was the change from baseline to the end of the maintenance phase (Week 16) in the combined analysis of the following measures of cognitive function:

- Controlled Oral Word Association (COWA) Tes ${ }^{10}$ of verbal fluency;

- Stroop Color-Word Interference Test ${ }^{11}$ of reading speed and interference;

- Digit Cancellation Test ${ }^{12}$ (a timed detection task that evaluates selective attention);

- Lafayette Grooved Pegboard Test (dominant hand $)^{13}$ of coordinated motor speed;

- Rey Auditory-Verbal Learning Test $(\text { RAVLT })^{14}$ of delayed recall of verbal memory;

- Symbol-Digit Modalities Test ${ }^{15}$ (a timed graphomotor coding task).

Change from baseline to the end of the maintenance phase for each individual cognitive test was also assessed, as were the percentages of patients whose results improved, did not change, or worsened on each cognitive test and the percentage of patients who were seizure free during the dose escalation phase and the maintenance phase. Other study end points were the maintenance dosage of study drug, the proportion of patients experiencing drug-related treatment-emergent adverse events, and the overall response to treatment at the end 
Table 1. Dosage escalation schedules for lamotrigine and topiramate.

\begin{tabular}{ccc} 
Study week & Lamotrigine dosage, $\mathrm{mg} / \mathrm{d}$ & 25 \\
1 & 50 & Topiramate dosage, $\mathrm{mg} / \mathrm{d}$ \\
2 & 50 & 50 \\
\hline 3 & 100 & 75 \\
4 & 100 & 100 \\
\hline 5 & 200 & 125 \\
6 & 300 & 150 \\
\hline 7 & 400 & 200 \\
$8-16$ & 500 & 300 \\
\hline
\end{tabular}

Patients were assigned randomly, in a 1:1 ratio, to receive either lamotrigine or topiramate.

Table 2. Baseline demographics and characteristics.

\begin{tabular}{lcc} 
& Patient characteristics & Lamotrigine (n=25) \\
Age, mean (SD), years & $57.7(8.9)$ & $56.2(7.0)$ \\
Sex, n. (\%) & & \\
$\quad$ Male & $15(60)$ & $14(54)$ \\
$\quad$ Female & $10(40)$ & $12(46)$ \\
\hline Age at first seizure, mean (SD), years & $37.3(18.5)^{*}$ & $31.1(19.4)$ \\
N. seizures per month, mean (SD) & $3.0(2.4)$ & $2.6(1.9)$ \\
\hline Seizure classification, ${ }^{*}$ n. (\%) & & \\
$\quad$ Simple partial & $9(36)$ & $13(50)$ \\
$\quad$ Complex partial & $23(92)$ & $24(92)$ \\
Secondarily generalized & $12(48)$ & $3(31)$ \\
$\quad$ Primary generalized tonic-clonic & $1(4)$ & $12(46)$ \\
AED use (most commonly used AEDs), n. (\%) & $9(36)$ & $14(54)$ \\
$\quad$ Carbamazepine & $16(64)$ & \\
Phenytoin &
\end{tabular}

$A E D$, antiepileptic drug. ${ }^{*}$ Age at first seizure was available for 24 patients in the lamotrigine group. ${ }^{*}$ Multiple seizure classifications are possible for the same patient.

Table 3. Cognitive function test results in study completers.

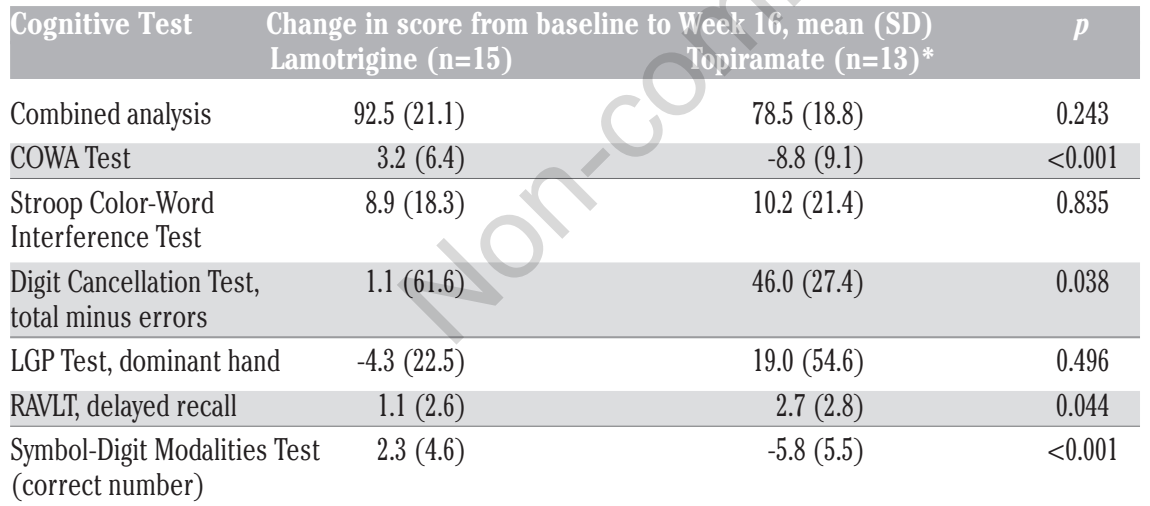

COWA, Controlled Oral Word Association; LGP, Lafayette Grooved Pegboard; RAVLT, Rey Auditory-Verbal Learning Test. * $n=12$ for the combined analysis, Digit Cancellation Test, and Symbol-Digit Modalities Test. For the LGP Test, a lower change score indicates better cognitive performance. For all other tests, higher change scores indicate better cognitive performance.

of the dose escalation phase and the end of the maintenance phase, as rated on a 7 point Likert-type scale. Using this scale, patients, family members, and clinicians rated overall response to treatment since beginning lamotrigine/topiramate therapy as "very much improved," "much improved," "minimally improved," "no change," "minimally worsened," "much worsened," or "very much worsened." Family member assessments, conducted only when a family member was available, were not collected for all patients.

\section{Statistical methods}

The primary end point of the study (i.e. change from baseline to the end of the mainte- nance phase in combined cognitive test results) was analyzed via the 0 'Brien test ${ }^{16}$ and analysis of variance (ANOVA). Ranks were assigned to individual test results for each patient, with higher scores representing better cognitive function. A total rank was calculated for each patient by adding the ranks of the individual tests, and ANOVA was used to test for differences between the lamotrigine and topiramate groups in patient total rankings.

Differences between treatment groups for individual cognitive tests were analyzed via analysis of covariance (ANCOVA); the change in score from baseline to the end of the maintenance phase was the dependent variable, treatment and age category were predictor variables, and screening score was a covariate. The incidence of seizure freedom was compared between treatment groups with the Fisher's exact test. The proportion of patients citing cognitive decline as the primary reason for premature study discontinuation was compared between treatment groups with a $\chi^{2}$ test. For all tests, values of $p \leq 0.05$ were considered significant. All other study end points were summarized with descriptive statistics. For analysis of the patient, family, and clinician assessment data, the "very much", "much", and "minimally improved" responses were combined into one category ("improved"), and the "very much", "much", and "minimally worsened" responses were combined into one category ("worsened"). Cognitive function, seizure freedom, and patient, family, and clinician assessment data were evaluated for patients who completed the study. Adverse event data were evaluated for all patients.

\section{Results}

\section{Demographics, baseline character- istics, and patient disposition}

Fifty-one patients (lamotrigine, $\mathrm{n}=25$; topiramate, $n=26$ ) were included in the analysis. Baseline demographics and patient characteristics are shown in Table 2. Fifteen patients $(60 \%)$ in the lamotrigine group and 13 patients (50\%) in the topiramate group completed the trial. Reasons for premature study discontinuation included adverse event(s) (lamotrigine, $n=7$; topiramate, $\mathrm{n}=9$ ), withdrawal of consent (lamotrigine, $\mathrm{n}=1$; topiramate, $\mathrm{n}=1$ ), lost to follow-up (lamotrigine, $n=1$; topiramate, $n=0$ ), protocol violation (lamotrigine, $n=0$; topiramate, $n=1$ ), and other reasons (lamotrigine, $\mathrm{n}=1$; topiramate, $\mathrm{n}=2$ ). The median maintenance dosage was $500.0 \mathrm{mg} / \mathrm{d}$ (range, 314.6-500.0 $\mathrm{mg} / \mathrm{d}$ ) for lamotrigine and $300.0 \mathrm{mg} / \mathrm{d}$ (range, $262.5-300.0 \mathrm{mg} / \mathrm{d}$ ) for topiramate. 
Table 4. Proportion of patients improving, having no change, or worsening from baseline to Week 16 for each cognitive test.

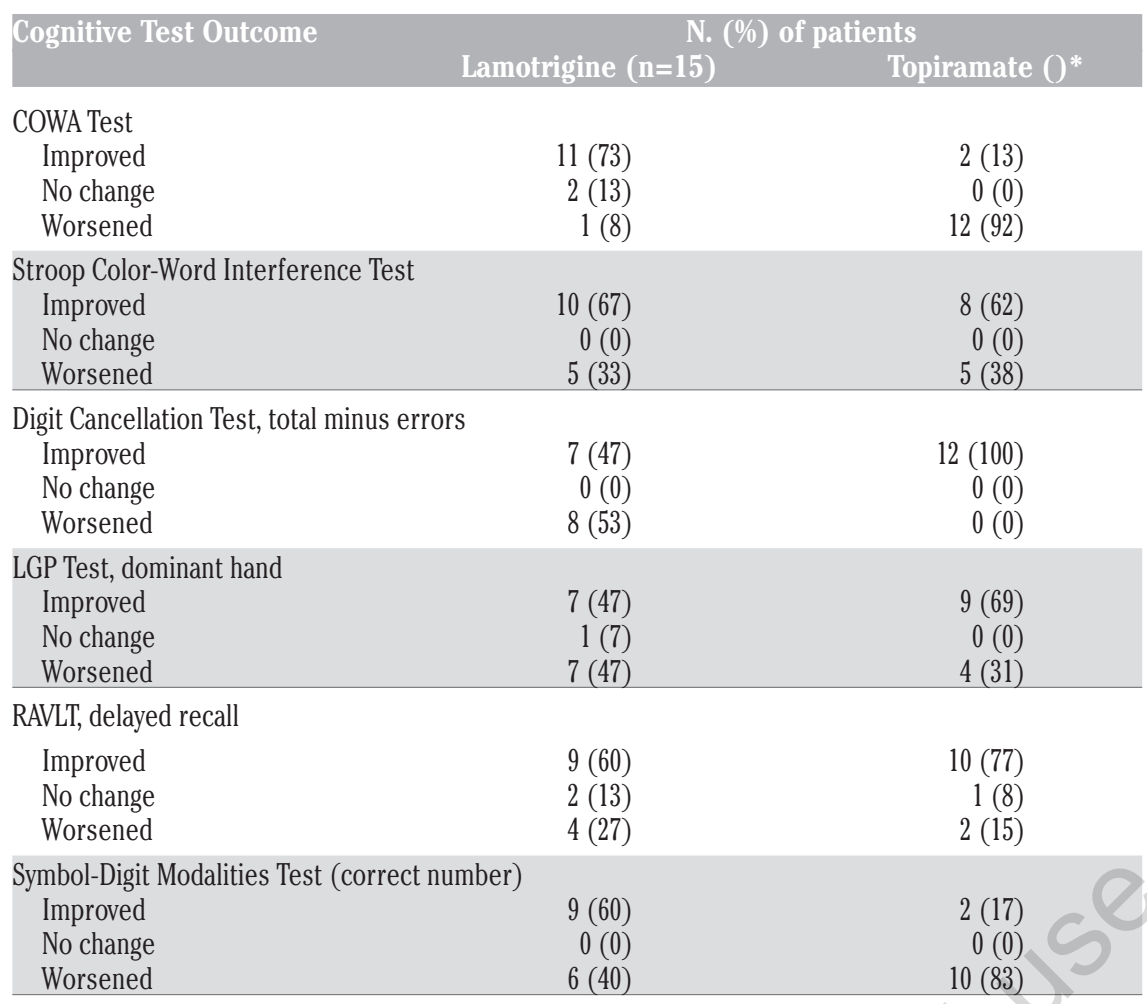

COWA, Controlled Oral Word Association; LGP, Lafayette Grooved Pegboard; RAVLT, Rey Auditory-Verbal Learning Test. ${ }^{n} n=12$ for the Digit Cancellation Test and Symbol-Digit Modalities Test.

Table 5. Seizure freedom in study completers.

\begin{tabular}{lccc} 
Study phase & \multicolumn{2}{c}{$\mathbf{N}(\mathbf{\%})$ of patients seizure free } & $p$ \\
& Lamotrigine $(\mathbf{n = 1 5 )}$ & Topiramate $(\mathbf{n = 1 3 )})$ & \\
Dose escalation & $6(40)$ & $5(38)$ & 1.00 \\
Maintenance & $6(40)$ & $6(46)$ & 1.00 \\
\hline Entire treatment period & $5(33)$ & $4(31)$ & 1.00 \\
\hline
\end{tabular}

Table 6. Patient, family, and clinician assessments of overall treatment response in subjects who completed the study.

\begin{tabular}{lcc}
\hline Assessment & Lamotrigine, $\mathrm{n} .(\%)$ & Topiramate, $\mathrm{n} .(\%)$ \\
Patient & $\mathrm{n}=15$ & $\mathrm{n}=12$ \\
Improved & $10(67)$ & $8(67)$ \\
No change & $3(20)$ & $4(33)$ \\
Worsened & $2(13)$ & $0(0)$ \\
Family & $\mathrm{n}=9$ & $\mathrm{n}=9$ \\
Improved & $5(56)$ & $4(44)$ \\
No change & $3(33)$ & $3(33)$ \\
Worsened & $1(11)$ & $2(22)$ \\
Clinician & $\mathrm{n}=15$ & $\mathrm{n}=13$ \\
Improved & $11(73)$ & $10(77)$ \\
No change & $3(20)$ & $2(15)$ \\
Worsened & $1(7)$ & $1(8)$ \\
\hline
\end{tabular}

\section{Cognitive function}

For the primary end point (i.e., combined results of cognitive tests), no significant dif- in mean change from baseline to Week 16 (Table 3). However, significant differences between treatment groups were noted for ference between treatment groups was noted some individual cognitive tests. Changes from baseline on the COWA Test and the SymbolDigit Modalities Test were significantly better for lamotrigine-treated patients than for topiramate-treated patients (Table 3). However, changes from baseline on the Digit Cancellation Test and the RAVLT delayed recall test were significantly better for the topiramate group than for the lamotrigine group. Table 4 shows the proportion of patients in each treatment group who improved, did not change, or worsened for each of the cognitive tests.

\section{Seizure freedom and overall assessments}

No significant differences were noted between treatment groups in the proportion of patients who were seizure free during the dose escalation phase, the maintenance phase, or the entire treatment period (Table 5). Patient, family, and clinician assessments of overall treatment response were generally similar between the treatment groups (Table 6).

\section{Adverse events}

Study drug-related treatment-emergent adverse events that were reported in $\geq 5 \%$ of patients in either treatment group and adverse events that led to study discontinuation in $\geq 5 \%$ of patients in either treatment group are shown in Table 7 . The proportion of patients citing cognitive decline as the primary reason for study discontinuation was not significantly different between treatment groups (lamotrigine, $0 \%$; topiramate, $15 \%$ ).

\section{Discussion}

Aging is associated with physiological changes that can alter AED metabolism and pharmacokinetics ${ }^{2,5}$ often resulting in higher plasma concentrations of AEDs. In particular, plasma concentrations of unbound AEDs tend to be higher in older adults than in younger adults, ${ }^{5}$ which may increase cognitive and other side effects at dosages that are usually effective and well-tolerated in younger adults. In addition, older patients tend to take more medications for other medical illnesses, which may cause cognitive side effects directly or interact with their AEDs. Therefore, the conventional assumption is that cognitive effects of AEDs are amplified in elderly patients when compared with younger adults, especially with AEDs that more frequently cause cognitive side effects in younger patients. Results from previous studies have suggested that overall topiramate is associated with a greater degree of cognitive impairment than is lamotrigine. ${ }^{1,17}$ 
Table 7. Study drug-related treatment-emergent adverse events $(\geq 5 \%$ of patients in either treatment group) and adverse events leading to study discontinuation ( $\geq 5 \%$ of patients in either treatment group).

\begin{tabular}{lrr} 
Adverse Event & N. (\%) of patients \\
& Lamotrigine $(\mathbf{n = 2 5})$ & Topiramate (n=26) \\
Events in $\geq 5 \%$ of patients in either treatment group & & \\
Any adverse event & $14(56)$ & $14(54)$ \\
Balance disorder & $2(8)$ & $1(4)$ \\
Coordination abnormal & $2(8)$ & $0(0)$ \\
Dizziness & $3(12)$ & $1(4)$ \\
Fatigue & $2(8)$ & $2(8)$ \\
Insomnia & $2(8)$ & $1(4)$ \\
Irritability & $2(8)$ & $1(4)$ \\
Nausea & $4(16)$ & $1(4)$ \\
Rash & $2(8)$ & $0(0)$ \\
Somnolence & $0(0)$ & $3(12)$ \\
Events leading to study discontinuation in $\geq 5 \%$ of patients in & either treatment group & \\
Any adverse event & $7(28)$ & $9(35)$ \\
Balance disorder & $2(8)$ & $0(0)$ \\
Dizziness & $2(8)$ & $0(0)$ \\
Nausea & $2(8)$ & $0(0)$ \\
\hline Same &
\end{tabular}

Some patients reported more than one adverse event as reason for study discontinuation.

${ }^{19}$ However, one recent study reported that topiramate at $50 \mathrm{mg}$ or $200 \mathrm{mg} /$ day was actually well tolerated in the elderly population. ${ }^{20}$

Our study is the first to specifically compare the cognitive effects of lamotrigine and topiramate in older patients. Although the small sample size for this study precludes definitive conclusions on efficacy, our patient population tolerated both lamotrigine and topiramate relatively well. In fact, no significant difference in overall cognitive test scores was noted in this analysis of older patients, even though the previous study had shown greater cognitive impairment associated with topiramate therapy than lamotrigine. ${ }^{9}$ Nonetheless, when individual test results were analyzed separately, significantly better scores were noted for lamotrigine-treated patients than for topiramate-treated patients on the COWA Test and the Symbol-Digit Modalities Test. On the other hand, topiramate-treated patients had significantly better scores than did lamotrigine-treated patients on the Digit Cancellation Test and the RAVLT delayed recall test. Similarly, substantially greater proportions of patients in the lamotrigine group than in the topiramate group improved from baseline to Week 16 on the COWA Test and the Symbol-Digit Modalities Test, whereas a substantially greater proportion of patients in the topiramate group than in the lamotrigine group improved on the Digit Cancellation Test. In comparison to the lamotrigine group, the topiramate group had a larger proportion of patients who improved from baseline to Week 16 on the RAVLT delayed recall test and smaller proportions of patients who had no change or worsened. Notably, among patients who prematurely terminated the study, cognitive decline was the primary reason for study discontinuation for more patients in the topiramate group (2 of $13.15 \%$ ) than in the lamotrigine group (0 of $10)$. The difference between groups was not statistically significant, however.

One important limitation of the current study is the small number of patients who are 50 years old or older. The $\geq 50$ year age cut-off differs from more commonly used age cut-offs for "elderly" patients (eg, 60 or 65 years). The age cut-off in the current analysis was mainly chosen to provide a larger sample size than would have been obtained with a more traditional age cut-off of 60 or 65 years. It is also important to note that the present analysis investigated the cognitive effects of lamotrigine versus topiramate as adjunctive therapy, and the results cannot necessarily be extrapolated to monotherapy.

It is not clear why older patients showed different tolerability profiles to younger patients, but this could possibly be due to their exposure to other concurrent medications, difference in dosing and pharmacokinetics, or simply due to the small sample size of our study. Clinicians should consider cognitive effects when prescribing AEDs, particularly for older patients who may already be experiencing some degree of cognitive decline ${ }^{2,3}$ and who may be generally more susceptible to cognitive effects of AEDs. ${ }^{1-4}$ Results from this study suggest that, in older patients, lamotrigine may have less of an impact on some areas of cognitive function (ie, verbal fluency and graphomotor speed), whereas topiramate may have less of an impact on other areas (ie, delayed recall of verbal memory and selective attention).

Although our study does not fully answer clinicians' questions about the relative tolerability in cognitive side effects of lamotrigine versus topiramate in older patients with epilepsy, it does demonstrate that different areas of cog- nitive function may be affected by these two medications. The results further support the need for evidence based practice rather than assumptions when counseling AED therapy for older patients regarding cognitive side effects. Future comparative study with a larger number of patients in a monotherapy trial with different AEDs would also be beneficial.

\section{References}

1. Martin R, Meador K, Turrentine L, et al. Comparative cognitive effects of carbamazepine and gabapentin in healthy senior adults. Epilepsia 2001;42:764-71.

2. Hirsch E, Schmitz B, Carreno M. Epilepsy, antiepileptic drugs (AEDs) and cognition. Acta Neurol Scand 2003;108: 23-32.

3. Motamedi GK, Meador KJ. Antiepileptic drugs and memory. Epilepsy \& Behavior, 2004;5:435-9.

4. Ortinski P, Meador KJ. Cognitive side effects of antiepileptic drugs. Epilepsy \& Behavior 2004;5:60-5.

5. Perucca E, Berlowitz D, Birnbaum A, et al. Pharmacological and clinical aspects of antiepileptic drug use in the elderly. Epilepsy Res 2006;68:49-63.

6. Aldenkamp AP, Baker G. A systematic review of the effects of lamotrigine on cognitive function and quality of life. Epilepsy \& Behavior 2001;2:85-91.

7. Huang CW, Pai MC, Tsai JJ. Comparative cognitive effects of levetiracetam and topiramate in intractable epilepsy. Psychiatry Clin Neurosc 2008;62:548-53.

8. Chung S, Wang N, Hank N. Comparative retention rates and long-term tolerability of new antiepileptic drugs. Seizure 2007;16:296-304.

9. Blum D, Meader K, Biton V, et al. Cognitive effects of lamotrigine compared with topiramate in patients with epilepsy. Neurology 2006;67:400-6.

10. Ruff RM, Light RH, Parker SB, Levin HS. Benton Controlled Oral Word Association Test: reliability and updated norms. Arch Clin Neuropsychol 1996;11:329-38.

11. Jensen AR, Rohwer WD. The Stroop ColorWord Test: a review. Acta Psychologica (Amst) 1966;25:36-93.

12. Della Sala S, Laiacona M, Spinnler H, Ubezio, C. A cancellation test: its reliability in assessing attentional deficits in Alzheimer's disease. Psychological Med 1992;22:885-901.

13. Silbert BS, Maruff P, Evered LA, et al. Detection of cognitive decline after coronary surgery: a comparison of computerized and conventional tests. Br J Anaesth 2004:92:814-20.

14. Mungas D. Differential clinical sensitivity 
of specific parameters of the Rey AuditoryVerbal Learning Test. J Consulting \& Clin Psychol 1983;51: 848-55.

15. Sheridan LK, Fitzgerald HE, Adams KM, et al. Normative Symbol Digit Modalities Test performance in a community-based sample. Arch Clin Neuropsychol 2006;21:23-8.

16. O'Brien PC. Procedures for comparing samples with multiple endpoints. Biometrics 1984;40:1079-87.
17. Kockelmann E, Elger CE, Helmstaedter C. Cognitive profile of topiramate as compared with lamotrigine in epilepsy patients on antiepileptic drug polytherapy: relationships to blood serum levels and comedication. Epilepsy \& Behavior 2004;5: 716-21.

18. Meador KJ, Loring DW, Vahle VJ, et al. Cognitive and behavioral effects of lamotrigine and topiramate in healthy volun- teers. Neurology 2005;64:2108-14.

19. Smith ME, Gevins, A McEvoy, et al. Distinct cognitive neurophysiologic profiles for lamotrigine and topiramate. Epilepsia 2006;47:695-703.

20. Ramsay RE, Uthman B, Pryor FM, et al. Topiramate in older patients with partialonset seizures: a pilot double-blind, dosecomparison study. Epilepsia 2008;49:1180-5. 